\title{
Rhinusinusitis
}

\section{Kochsalzlösung unterstützt die Therapie}

— Die spezifische Therapie bei chronischer Rhinosinusitis lässt sich durch natürliche Arzneien unterstützen: So kann laut aktueller Leitlinie der Deutschen Gesellschaft für Hals-Nasen-Ohren-Heilkunde, Kopfund Hals-Chirurgie die lokale Anwendung von hypertonen Salzlösungen eine Linderung der Beschwerden bewirken. Durch den osmotischen Effekt schwellen die Nasenschleimhäute ab, das Nasensekret verflüssigt sich. Dadurch werde die mukoziliäre Clearance verbessert, teilt das Unternehmen Bionorica SE mit.
Eine kontrollierte, prospektive Studie mit 211 Patienten habe bei $2 x$ täglicher Anwendung einer hypertoner Salzlösung zur Reduktion der Beschwerden geführt. In einem Cochrane Review sei der Nutzen einer topischen Salzlösung als unterstützende Maßnahme bei chronischer Rhinosinusitis bestätigt worden.

Rinupret ${ }^{\circledR}$-Pflege-Nasenspray enthält außer natürlichem Meersalz in hypertoner Lösung (2,7\%-ig) Eukalyptusöl, das belebend wirkt, sowie pflegende Aloe vera. Der Nasenschleimhaut wird durch die Salzkonzentra- tion im Spray überschüssiges Wasser entzogen, die Schleimhäute schwellen ab. Die frei werdende Flüssigkeit löst zähes Sekret und Verkrustungen. Der Effekt ist lokal und unterstützt die Selbstreinigungs- und Abwehrfunktion der Nasenschleimhaut auch bei längerfristiger Anwendung. Das gut verträgliche Präparat kann bei Erwachsenen und Kindern ab zwei Jahren angewendet werden. Es ist zur begleitenden Behandlung bei Entzündungen der Nasenschleimhaut mit verstopfter Nase oder Schnupfen geeignet. - Red.

\section{Leben mit Typ-1-Diabetes \\ Ratgeber mit Therapie-Update neu aufgelegt}

- Mit der 2. Auflage des Taschenbuches „Diabetes bei Kindern" steht ein aktueller Ratgeber zur Unterstützung der Eltern von Typ-1-Diabetikern zur Verfügung (TRIAS Verlag, Stuttgart 2012). Er liefert eine realistische Darstellung der Situation pädiatrischer Patienten mit Typ-1-Diabetes und ist bei Eltern wie Kinder-Diabetologen gleichermaßen beliebt, berichtete Dipl.-Psych. Béla Bartus, Stuttgart. Die mit Unterstützung der Firma Lilly realisierte Überarbei- tung war erforderlich, da sich die Therapie des Typ-1-Diabetes verändert hat. So wurde die Insulintherapie deutlich intensiviert, entweder in Form der intensivierten konventionellen Insulintherapie (ICT) oder als Pumpentherapie, berichtete Dr. Martin Holder, Stuttgart. Als Fortschritt wertete er insbesondere die Einführung kurz wirksamer Analoginsuline wie Insulin lispro (Humalog ${ }^{\oplus}$ ), die 2010 bereits von rund $60 \%$ der pädiatrischen Patienten täglich ge- spritzt wurden. Gerade bei Kindern, deren Nahrungsaufnahme oft schlecht planbar ist, haben diese Insuline Vorteile. Dazu zählen der Wegfall des Spritz-Ess-Abstandes oder die mögliche Gabe erst nach der Mahlzeit, sodass die Dosis genau auf die Nahrungsmenge abgestimmt werden kann.

- Dr. Katharina Arnheim Quelle: Pressegespräch „Typ 1 Diabetes: die Kleinsten benötigen die größte Flexibilität", Stuttgart, Mai 2012 (Veranstalter: Lilly) 